\title{
Uniform Boundedness Principle
}

\author{
Hideki Sakurai \\ Shinshu University \\ Nagano, Japan
}

\author{
Hisayoshi Kunimune \\ Shinshu University \\ Nagano, Japan
}

\author{
Yasunari Shidama \\ Shinshu University \\ Nagano, Japan
}

Summary. In this article at first, we proved the lemma of the inferior limit and the superior limit. Next, we proved the Baire category theorem (Banach space version) [20], [9], [3], quoted it and proved the uniform boundedness principle. Moreover, the proof of the Banach-Steinhaus theorem is added.

MML identifier: LOPBAN_5, version: $\underline{7.8 .05 \quad 4.89 .993}$

The articles [17], [18], [15], [12], [19], [1], [21], [5], [8], [7], [16], [10], [6], [13], [4], $[2],[14]$, and [11] provide the terminology and notation for this paper.

\section{Uniform Boundedness PRInCIPLE}

The following two propositions are true:

(1) For every sequence $s_{1}$ of real numbers and for every real number $r$ such that $s_{1}$ is bounded and $0 \leq r$ holds $\liminf \left(r s_{1}\right)=r \cdot \liminf s_{1}$.

(2) For every sequence $s_{1}$ of real numbers and for every real number $r$ such that $s_{1}$ is bounded and $0 \leq r$ holds $\lim \sup \left(r s_{1}\right)=r \cdot \lim \sup s_{1}$.

Let $X$ be a real Banach space. One can verify that MetricSpaceNorm $X$ is complete.

Let $X$ be a real Banach space, let $x_{0}$ be a point of $X$, and let $r$ be a real number. The functor Ball $\left(x_{0}, r\right)$ yielding a subset of $X$ is defined as follows:

(Def. 1) $\operatorname{Ball}\left(x_{0}, r\right)=\left\{x ; x\right.$ ranges over points of $\left.X:\left\|x_{0}-x\right\|<r\right\}$.

The following propositions are true:

(3) Let $X$ be a real Banach space and $Y$ be a sequence of subsets of $X$. Suppose $\bigcup \operatorname{rng} Y=$ the carrier of $X$ and for every element $n$ of $\mathbb{N}$ holds $Y(n)$ is closed. Then there exists an element $n_{0}$ of $\mathbb{N}$ and there exists 
a real number $r$ and there exists a point $x_{0}$ of $X$ such that $0<r$ and $\operatorname{Ball}\left(x_{0}, r\right) \subseteq Y\left(n_{0}\right)$.

(4) Let $X, Y$ be real normed spaces and $f$ be a bounded linear operator from $X$ into $Y$. Then

(i) $\quad f$ is Lipschitzian on the carrier of $X$ and continuous on the carrier of $X$, and

(ii) for every point $x$ of $X$ holds $f$ is continuous in $x$.

(5) Let $X$ be a real Banach space, $Y$ be a real normed space, and $T$ be a subset of the real norm space of bounded linear operators from $X$ into $Y$. Suppose that for every point $x$ of $X$ there exists a real number $K$ such that $0 \leq K$ and for every point $f$ of the real norm space of bounded linear operators from $X$ into $Y$ such that $f \in T$ holds $\|f(x)\| \leq K$. Then there exists a real number $L$ such that

(i) $0 \leq L$, and

(ii) for every point $f$ of the real norm space of bounded linear operators from $X$ into $Y$ such that $f \in T$ holds $\|f\| \leq L$.

Let $X, Y$ be real normed spaces, let $H$ be a function from $\mathbb{N}$ into the carrier of the real norm space of bounded linear operators from $X$ into $Y$, and let $x$ be a point of $X$. The functor $H \# x$ yields a sequence of $Y$ and is defined by:

(Def. 2) For every element $n$ of $\mathbb{N}$ holds $(H \# x)(n)=H(n)(x)$.

The following proposition is true

(6) Let $X$ be a real Banach space, $Y$ be a real normed space, $v_{1}$ be a sequence of the real norm space of bounded linear operators from $X$ into $Y$, and $t_{1}$ be a function from $X$ into $Y$. Suppose that for every point $x$ of $X$ holds $v_{1} \# x$ is convergent and $t_{1}(x)=\lim \left(v_{1} \# x\right)$. Then

(i) $t_{1}$ is a bounded linear operator from $X$ into $Y$,

(ii) for every point $x$ of $X$ holds $\left\|t_{1}(x)\right\| \leq \liminf \left\|v_{1}\right\| \cdot\|x\|$, and

(iii) for every point $t_{2}$ of the real norm space of bounded linear operators from $X$ into $Y$ such that $t_{2}=t_{1}$ holds $\left\|t_{2}\right\| \leq \liminf \left\|v_{1}\right\|$.

\section{Banach-Steinhaus Theorem}

We now state two propositions:

(7) Let $X$ be a real Banach space, $X_{0}$ be a subset of LinearTopSpaceNorm $X$, $Y$ be a real Banach space, and $v_{1}$ be a sequence of the real norm space of bounded linear operators from $X$ into $Y$. Suppose that

(i) $X_{0}$ is dense,

(ii) for every point $x$ of $X$ such that $x \in X_{0}$ holds $v_{1} \# x$ is convergent, and

(iii) for every point $x$ of $X$ there exists a real number $K$ such that $0 \leq K$ and for every element $n$ of $\mathbb{N}$ holds $\left\|\left(v_{1} \# x\right)(n)\right\| \leq K$.

Let $x$ be a point of $X$. Then $v_{1} \# x$ is convergent. 
(8) Let $X, Y$ be real Banach spaces, $X_{0}$ be a subset of LinearTopSpaceNorm $X$, and $v_{1}$ be a sequence of the real norm space of bounded linear operators from $X$ into $Y$. Suppose that (i) $X_{0}$ is dense,

(ii) for every point $x$ of $X$ such that $x \in X_{0}$ holds $v_{1} \# x$ is convergent, and

(iii) for every point $x$ of $X$ there exists a real number $K$ such that $0 \leq K$ and for every element $n$ of $\mathbb{N}$ holds $\left\|\left(v_{1} \# x\right)(n)\right\| \leq K$.

Then there exists a point $t_{1}$ of the real norm space of bounded linear operators from $X$ into $Y$ such that for every point $x$ of $X$ holds $v_{1} \# x$ is convergent and $t_{1}(x)=\lim \left(v_{1} \# x\right)$ and $\left\|t_{1}(x)\right\| \leq \liminf \left\|v_{1}\right\| \cdot\|x\|$ and $\left\|t_{1}\right\| \leq \liminf \left\|v_{1}\right\|$.

\section{REFERENCES}

[1] Grzegorz Bancerek. The ordinal numbers. Formalized Mathematics, 1(1):91-96, 1990.

[2] Czesław Byliński. Introduction to real linear topological spaces. Formalized Mathematics, 13(1):99-107, 2005.

[3] N. J. Dunford and T. Schwartz. Linear operators I. Interscience Publ., 1958.

[4] Noboru Endou, Yasunari Shidama, and Katsumasa Okamura. Baire's category theorem and some spaces generated from real normed space. Formalized Mathematics, 14(4):213$219,2006$.

[5] Krzysztof Hryniewiecki. Basic properties of real numbers. Formalized Mathematics, 1(1):35-40, 1990.

[6] Stanisława Kanas, Adam Lecko, and Mariusz Startek. Metric spaces. Formalized Mathematics, 1(3):607-610, 1990.

[7] Jarosław Kotowicz. Convergent sequences and the limit of sequences. Formalized Mathematics, 1(2):273-275, 1990.

[8] Jarosław Kotowicz. Real sequences and basic operations on them. Formalized Mathematics, 1(2):269-272, 1990.

[9] Isao Miyadera. Functional Analysis. Riko-Gaku-Sya, 1972.

[10] Andrzej Nędzusiak. $\sigma$-fields and probability. Formalized Mathematics, 1(2):401-407, 1990.

[11] Takaya Nishiyama, Keiji Ohkubo, and Yasunari Shidama. The continuous functions on normed linear spaces. Formalized Mathematics, 12(3):269-275, 2004.

[12] Beata Padlewska and Agata Darmochwał. Topological spaces and continuous functions. Formalized Mathematics, 1(1):223-230, 1990.

[13] Jan Popiołek. Real normed space. Formalized Mathematics, 2(1):111-115, 1991.

[14] Yasunari Shidama. Banach space of bounded linear operators. Formalized Mathematics, 12(1):39-48, 2004.

[15] Andrzej Trybulec. Binary operations applied to functions. Formalized Mathematics, 1(2):329-334, 1990.

[16] Wojciech A. Trybulec. Vectors in real linear space. Formalized Mathematics, 1(2):291-296, 1990.

17] Zinaida Trybulec. Properties of subsets. Formalized Mathematics, 1(1):67-71, 1990.

[18] Edmund Woronowicz. Relations and their basic properties. Formalized Mathematics, 1(1):73-83, 1990.

[19] Mirosław Wysocki and Agata Darmochwał. Subsets of topological spaces. Formalized Mathematics, 1(1):231-237, 1990.

[20] Kosaku Yoshida. Functional Analysis. Springer, 1980.

[21] Bo Zhang, Hiroshi Yamazaki, and Yatsuka Nakamura. Inferior limit and superior limit of sequences of real numbers. Formalized Mathematics, 13(3):375-381, 2005. 